\title{
Antenatal hydronephrosis: changing concepts in diagnosis and subsequent management
}

\author{
A.A. SHOKEIR and R.J.M. NIJMAN* \\ Urology \& Nephrology Center, Mansoura University, Mansoura, Egypt, and *Department of Paediatric Urology, Sophia Children's \\ Hospital, Eramus University, Rotterdam, The Netherlands
}

\section{Introduction}

Because of the increasing use of ultrasonography (US) for fetal-maternal screening, the number of fetuses identified with hydronephrosis has increased markedly. The appropriate prenatal care of the fetus diagnosed with such a condition, and the postnatal evaluation and treatment, are not always evident. Guidelines for the evaluation of this finding are outlined in the present review.

\section{Incidence}

The overall incidence of genitourinary abnormalities during routine prenatal US screening is 2-9 per 1000 births [1-5], with a male to female ratio of $2: 1$ [3]. The spectrum of genitourinary abnormalities detected in utero is extremely varied; most affected fetuses (50-87\%) have hydronephrosis [6-8]. The remaining anomalies include multicystic dysplastic kidney, autosomal recessive polycystic kidney disease, renal agenesis and dysplasia. Other abnormalities that have been described include bladder exstrophy, adrenal hyperplasia, imperforate anus, cloacal abnormalities, neuroblastoma, mesoblastic nephroma and genital abnormalities [6].

The accuracy of the prenatal diagnosis of hydronephrosis has been compared with postnatal evaluations and a false-positive diagnostic error rate of $9-22 \%$ is reported $[9,10]$. This overestimation may be because there are no accepted criteria for the diagnosis of hydronephrosis, and in utero resolution during fetal development [11].

\section{Natural history}

Antenatal hydronephrosis is mostly a physiological state which will resolve either before the end of pregnancy or within the first year of life. An explanation for spontaneous resolution of hydronephrosis is the transitional (antenatal to postnatal) renal physiology and histology/anatomy of the developing ureter. Fetal urine flow is 4-6 times greater before than after delivery because of differences in renovascular resistance, GFR and concentrating ability. This high outflow might contribute to ureteric dilatation in the absence of functionally significant obstruction, as is occasionally seen with diabetes insipidus [12]. Another contributing factor is the increased compliance of the fetal ureter. Increased compliance has been implicated in the tortuous dilatations seen with distal obstructions in the newborn, which contrast with the 'pipe stem' of later life. These changes may result from differences in the deposition and orientation of elastin, collagen and other matrices, which occur at various rates in the developing fetal ureter [13]. Finally, partial or transient anatomical or functional obstructions that spontaneously improve with postnatal development could contribute to proximal dilatation. Persistent fetal ureteric folds or delays in the development of normal peristalsis may represent such transient obstructions [12].

Most antenatal hydronephrosis that is confirmed postnatally, even with bilateral involvement, is mild or moderate as a consequence of partial obstruction during embryological development. The natural history of mild and moderate degrees of partial ureteric obstruction has been studied in neonatal rats. Josephson et al. [14] created mild to moderate partial obstruction and observed a decrease in renal blood flow (RBF), GFR and potassium excretion that approached $30 \%$, and was largely compensated by an increase in the contralateral kidney. The obstruction in the experimental model must be relieved early to reverse the loss of glomerular function.

The upper urinary tract in prenatally detected hydronephrosis is rarely completely obstructed. Such rare cases will result in a nonfunctioning kidney with severe cystic dysplasia of the nephrogenic tissue [7]. The outcome of these lesions is related to the occurrence of nonrenal congenital anomalies, which is much higher in those infants who ultimately die in the perinatal period. The strongest indicators of a poor prognosis in such cases include early onset of severe renal parenchymal changes, 
oligohydramnios, severe nonrenal congenital anomalies and bladder outlet obstruction [11]. When severe obstruction is produced experimentally, there is a rapid and progressive $80-90 \%$ decrease in RBF and GFR, which may not be recoverable $[15,16]$. Tubular function, such as potassium and phosphate excretion, is also significantly decreased. Experimentally, when obstruction in the neonatal period was sufficient to lead to a measured decrease in renal function, some of this was not recoverable by relieving the obstruction, whereas similar obstruction in the mature kidney produced reversible changes [16]. With complete unilateral ureteric obstruction (UUO) in neonatal rats, Chevalier et al. [17] recently showed that growth of the developing kidney decreased linearly with the duration of ureteric obstruction. Adaptive growth of the intact kidney exactly counterbalances the decreased growth rate of the obstructed kidney. These results suggest that to optimally preserve renal mass in the developing kidney with complete UUO, the obstruction should be relieved as soon as possible.

\section{Prenatal evaluation}

The ability of prenatal US to detect fetal urinary tract abnormalities is widely appreciated. Despite its wide application, its continued use as a screening procedure is still debated [18]. However, there may be specific historical information or maternal physical findings that may prompt the use of US for maternal-fetal monitoring. Known genetic or familial abnormalities, e.g. polycystic kidney disease or renal agenesis, could be one such indication [11]. Abnormal uterine fundal height for expected gestational age may be related to fetal genitourinary abnormalities. Polyhydramnios can be associated with unilateral hydronephrosis [19] and oligohydramnios can be caused by renal dysgenesis/ agenesis or severe urinary tract obstruction [20]. Elevated maternal AFP has also been associated with fetal uropathies [21].

When characterizing hydronephrosis detected in utero, the degree of dilatation must be correlated with the age of gestation at diagnosis, because it has been shown to vary during gestation. Several studies have evaluated the threshold of fetal hydronephrosis resulting in clinically significant neonatal disease $[6,11,22]$. In most, there are persistent postnatal renal abnormalities when the anteroposterior diameter (APD) of the fetal renal pelvis measures $>6 \mathrm{~mm}$ at $<20$ weeks, $>8 \mathrm{~mm}$ at 20-30weeks and $>10 \mathrm{~mm}$ at $>30$ weeks of gestation.

The degree of antenatal hydronephrosis after week 20 of gestation has been divided into five grades by Grignon et al. [23]:
- grade I, the pelvic APD is $1 \mathrm{~cm}$ with normal calyces

- grade II, APD 1-1.5 cm with normal calyces

- grade III, APD $>1.5 \mathrm{~cm}$ with slight caliectasis

- grade IV, APD $>1.5 \mathrm{~cm}$ with moderate caliectasis

- grade V, APD $>1.5 \mathrm{~cm}$ with severe caliectasis and cortical atrophy

A simpler classification is:

- mild hydronephrosis with pelvic APD $\leqslant 1.5 \mathrm{~cm}$ and normal calyces

- moderate hydronephrosis with APD $>1.5 \mathrm{~cm}$ and caliectasis with no parenchymal atrophy

- severe hydronephrosis with APD $>1.5 \mathrm{~cm}$, caliectasis and cortical atrophy [8].

\section{Diagnostic categories}

It is important to characterize fetal hydronephrosis by its severity, laterality and its association with ureteric dilatation, renal parenchymal changes, and abnormalities of bladder size, thickness and emptying. The presence of concomitant oligohydramnios is important in determining the prognosis.

PUJ obstruction is by far the most common cause of antenatal hydronephrosis and is characterized by varying degrees of fetal renal pelvic dilatation, caliectasis, no visible ipsilateral ureter, normal bladder and normal amniotic fluid volume. When ureteric dilatation occurs with pelvicalyceal dilatation, the most common postnatal abnormalities found are VUR, megaureter, PUV, prunebelly syndrome and ureteric ectopia. Because ureteric dilatation is often not seen, many cases of generic hydronephrosis may be caused by VUR or megaureter. The postnatal diagnosis of reflux is reported in $25-35 \%$ of fetuses with nonspecific hydronephrosis [24-26]. The presence of significant hydroureteronephrosis, a large thin-walled bladder, and normal renal architecture and amniotic fluid volume in a male fetus most probably signifies massive reflux, which has been termed megacystis-megaureter association [11,27]. Duplication anomalies should be suspected when there is asymmetric dilatation of the ipsilateral upper collecting systems, or a ureterocele can be seen in the bladder. In many such cases the diagnosis is not made until after birth [28].

PUV in a male fetus is characterized by uni- or bilateral hydroureteronephrosis, a thick-walled, trabeculated and sometimes enlarged bladder, a dilated posterior urethra, renal parenchymal change, such as hyperechogenicity or (subcortical) cysts, and varying degrees of oligohydramnios. PUV may be confused with prune-belly syndrome, characterized by ureteric dilatation that is proportionally greater than expected for renal pelvic diameter, an enlarged bladder with evidence of a urachal abnormality, 
and the presence of a megalourethra. In most cases, the amniotic fluid volume is normal [11].

\section{Fetal intervention}

Once a fetal genitourinary abnormality is diagnosed, the management of the fetus is controversial. In most cases, if the diagnosis is made in the second trimester, additional US is carried out closer to term. In bilateral hydroureteronephrosis and an enlarged bladder, US is recommended every 4 weeks after the initial diagnosis. Other more invasive diagnostic procedures to assess the overall status of the fetus include amniocentesis, per umbilical blood sampling (PUBS), and chorionic villous sampling. PUBS has become a standard method for rapid fetal sampling as early as 14-16 weeks of gestation, whereas chorionic villous sampling has the advantage of allowing chromosomal analysis at $8-10$ weeks [11].

The ability to analyse renal function and predict future renal reserve remains a difficult dilemma. The US finding of hyperechogenic fetal kidneys was a nonspecific marker for postnatal renal US or functional abnormalities [29]. The use of fetal bladder aspiration, and observation of refilling and examination of fetal urine is the most helpful guide to potential functional reserve. Numerous urinary electrolytes and proteins have been suggested as markers to predict the presence of severe renal injury in fetuses with obstructive uropathy, but without complete success. Of these, the most useful are albumin, B2-microglobulin, sodium, chloride and osmolality. Prognostic features for good renal function include fetal urinary sodium $<100 \mathrm{mmol} / \mathrm{L}$, chloride of $<90 \mathrm{mmol} / \mathrm{L}$, and an osmolality of $<210 \mathrm{mOsm} / \mathrm{kg}$. Vates et al. [30] evaluated the use of urinary EGF as a predictive marker of such injury. They found that fetuses with a good outcome had a trend toward higher EGF levels (mean $19.1 \mathrm{ng} / \mathrm{mL}$ ) in later gestation, compared with a decline (mean $6.5 \mathrm{ng} /$ $\mathrm{mL}$ ) in those with a poor outcome.

Elective termination of pregnancy is available up to about 23 weeks of gestation. Most major system defects can be detected if the gestational US is performed at 16-20 weeks. If severe abnormalities are detected then this option should be presented [11].

Prenatal intervention is indicated in a minority of cases of prenatally detected hydronephrosis. In such cases, there should be intervention only at an experienced institution under approved protocols [7]. The clinical setting in which fetal intervention involving chronic bladder drainage appears to be most appropriate is the male fetus in the second trimester who presents with severe bilateral hydroureteronephrosis, reasonable fetal urinary indicators, and progressive oligohydramnios. The rationale for fetal urinary drainage is that with severe early obstruction both renal and pulmonary development are impaired. Theoretically, bypassing the obstructed urinary system by shunting urine directly into the amniotic cavity will decompress the kidneys enough to prevent irreversible damage and prevent the development of pulmonary hypoplasia associated with oligohydramnios [11]. Successful percutaneous ureterostomy and vesicostomy has been recorded in a few patients [31]; no data are available from larger series on the outcome of kidney function after intrauterine intervention. However, complications such as obstruction or displacement of the shunt and bowel herniation along the needle tract have been reported. Moreover, fetal intervention may carry the risks of fetal injury, maternal infection, induction of early labour, miscarriage, risk to subsequent pregnancies, and inappropriate interventions for conditions that are not life-threatening.

\section{Postnatal management}

The initial assessment of a neonate with prenatally identified hydronephrosis will consist of confirmatory postnatal US usually 2-3 days after delivery, depending on the antenatal severity of dilatation and probable diagnosis. Unless hydroureteronephrosis is bilateral or a serious urological abnormality (e.g. PUV or obstruction of a solitary kidney) is suspected, further diagnostic evaluation can be deferred until one month of life. Welldocumented cases of pathology have been missed when US is used during the first few days of life because neonatal oliguria can mask a moderately obstructed lesion [32].

The importance of prophylactic antibiotics in infants with prenatally detected hydronephrosis that is confirmed postnatally has not been formally tested. Although it is expected that an infection with ureteric obstruction can cause significant renal damage, there are no published data available to suggest that antibiotic prophylaxis has a better outcome. Therefore, some authors recommend prophylactic antibiotics to all infants with prenatally detected hydronephrosis that is confirmed postnatally, while other institutions only administer antibiotics when the dilatation is severe (e.g. $>10 \mathrm{~mm}$ renal pelvis). Oral penicillin G $(20 \mathrm{kIU} /$ $\mathrm{kg})$, oral amoxicillin $(15 \mathrm{mg} / \mathrm{kg})$ or trimethoprim $(2 \mathrm{mg} /$ $\mathrm{kg}$ ) once a day are commonly used.

When the initial evaluation reveals bilateral hydronephrosis or unilateral hydronephrosis in a single functioning kidney, immediate US and VCUG are performed. If PUV is confirmed, then a resection or drainage is instituted. If VUR or an (obstructive) megaureter is found, then antibiotic prophylaxis is begun. When hydronephrosis is unilateral, or if bilateral and less marked, a diagnostic algorithm is used (Fig. 1). 


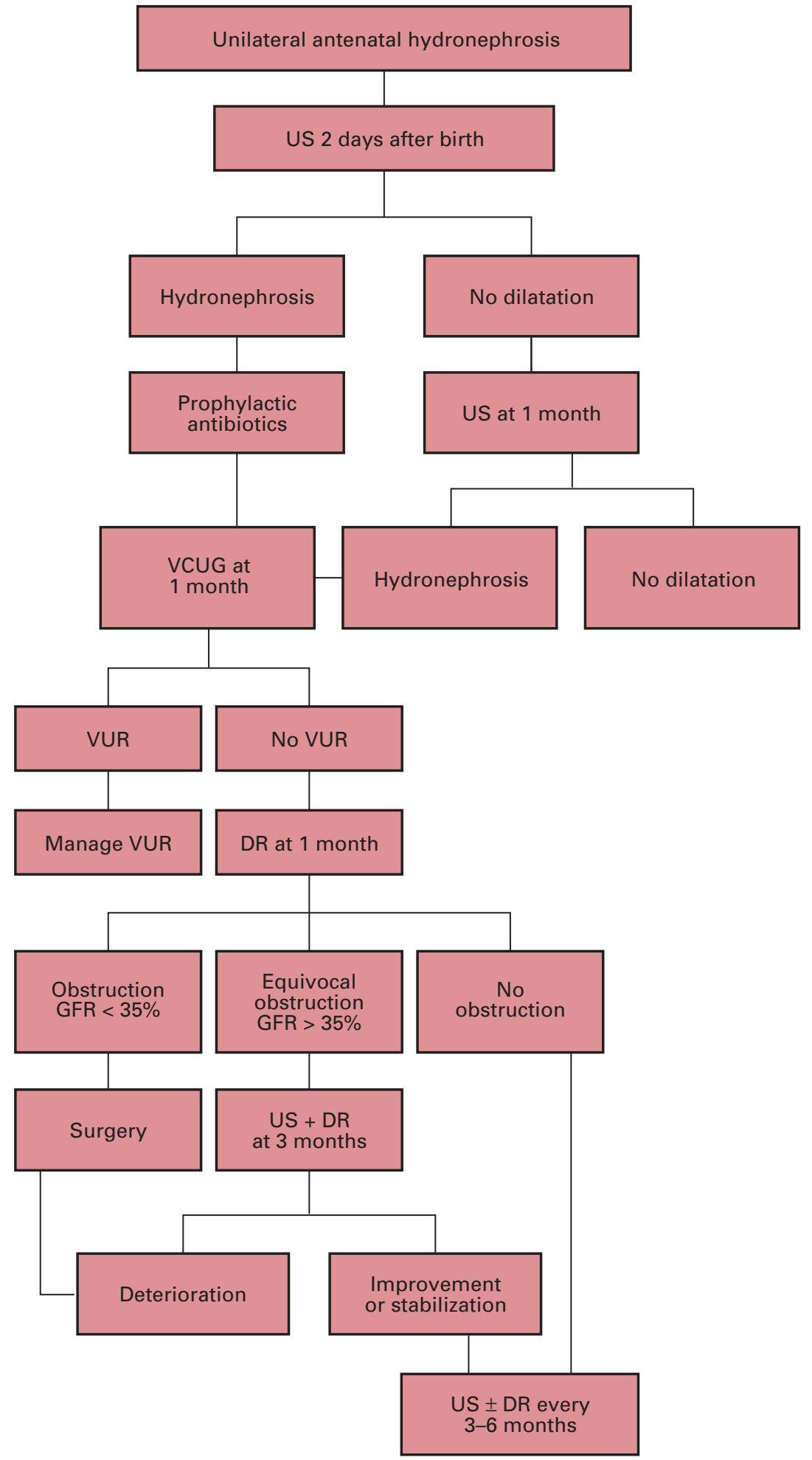

Fig. 1. An algorithm for neonatal evaluation of unilateral or mild bilateral hydronephrosis. US, ultrasonography; DR, diuretic renogram.

\section{Postnatal diagnosis of obstruction}

It took more than half a century for urologists to recognize that hydronephrosis is not necessarily equivalent to obstruction. Several studies showed that many neonatal kidneys with severe hydronephrosis are not biologically obstructed despite even a profound initial decrease in renal function. Further follow-up of these kidneys showed no deterioration of renal function, no progression of hydronephrosis and no evidence of 
contralateral compensatory renal hypertrophy. On the other hand, misdiagnosis of a truly obstructed kidney results in a serious deterioration of renal function that may be irreversible. Therefore, a continuing debate in the management of neonatal hydronephrosis is concerned with the definition of when significant obstruction is present. All of the standard diagnostic tests for assessing hydronephrosis in this age group, e.g. IVU, US, renogram and the Whitaker test, are inaccurate. The diagnosis in most cases is only possible by repeated investigations and changes of the variables during a longer follow-up.

\section{IVU and US}

In the neonate, reliance on the morphological appearance of a dilated renal pelvis from initial IVU or US is insufficient to diagnose obstruction. However, the detection of progressive hydronephrosis on further sequential studies could be an indicator of the presence of obstruction. Koff et al. [33] used US to measure the size of the normal kidney opposite a unilateral hydronephrotic kidney in infants, to determine if compensatory changes occurred and could be used as a diagnostic test for defining or excluding obstruction. They showed that normal kidneys opposite obstructed hydronephrotic kidneys become larger than normal for age. They suggested that by plotting serial measurements of normal renal length on a renal growth chart, the diagnosis of obstruction in newborn hydronephrosis can be facilitated and the clinical management of the patient improved. This view is supported by the recent experimental work of Chevalier et al. [17] who showed an exquisite sensitivity of renal counterbalance in complete UUO in the developing kidney of neonatal rats.

The potential usefulness of the resistive index (RI) obtained during Doppler ultrasonography (DUS) was described by Platt et al. in 1989 [34]. The noninvasive nature of the measurement of renal RI gives it considerable appeal in its potential application in children with obstructive uropathy. Although an RI of 0.70 is accepted by most investigators as an upper level of normal in the adult population, more work is needed to establish accepted standards of normal and abnormal for the paediatric renal RI. It has been shown that the RI is age-dependent, particularly in infants, and it is commonly $>0.70$ in healthy children in the first year of life $[35,36]$. A normal RI in this setting should be valuable, arguing against obstruction in the presence of a dilated collecting system. We have shown that infusion of normal saline and administration of frusemide significantly increased the accuracy of the RI in the diagnosis of obstructed kidneys in children [37,38]. The RI is considered as a noninvasive method for monitoring the dilated collecting system under observation.

\section{Diuretic renogram}

The post-frusemide drainage curve and the half-time drainage $\left(\mathrm{T}_{1 / 2}\right)$ are subject to so many variables that they are unacceptable as indicators of the presence of obstruction. The state of hydration, renal function, volume and contractility of the renal pelvis, patient position, bladder filling, timing and dose of diuretic administration and the type of radiopharmaceutical may all result in misinterpretation. Measurement of individual renal function with demarcation of a decrease in function has been adopted to determine the presence of obstruction. The critical value of individual renal function below which obstruction is considered varied among different studies. Values of $<30 \%$ [39], $<35 \%$ [40], or $<40 \%$ $[41,42]$ have been suggested (normal $=50 \pm 5 \%$ ). Having adopted this principle, the changes of transitional nephrology during the first weeks of life must be considered. It is well known that renal function improves rapidly in infancy, that the fastest rate of increase in GFR occurs within the first 12 weeks of life and that this rate of increase is inversely proportional to age [43]. The younger the patient, the more rapidly renal function improves as the kidney matures and ultimately attains adult levels of renal function. This age-related effect of renal maturation on improvement in renal function should not be misinterpreted as a true improvement in GFR after the surgical repair of suspected obstruction. Failure of expected improvement in renal function on further sequential radionuclide studies could be an indicator of the presence of obstruction.

One way to improve the quality of the diuretic renogram is to carry out the study under conditions of maximal diuresis. After an oral water load of $20 \mathrm{~mL} / \mathrm{kg}$ body weight, hypotonic saline $(\mathrm{NaCl} 0.45 \%$ with glucose $2.5 \%$ ) is administered intravenously over a 2 -h period in a dose of $2 \mathrm{~L} / 1.73 \mathrm{~m}^{2}$ body surface. Especially in cases of PUJ obstruction this seems to add significantly to the specificity and sensitivity of the test [44].

The extraction factor $(\mathrm{EF})$ is a component of the radionuclide renal scan that has been used by some investigators as a means of gauging clinically significant obstruction. The EF measures the percentage uptake of radionuclide by the kidneys $2-3 \mathrm{~min}$ after injection. The normal mean EF in the newborn is $1.5 \%$ by each kidney, with an increase to $2.5 \%$ occurring by the end of the first year of life. The correlation coefficient of the EF with GFR is $\approx 0.92$. If renal function is normal by $\mathrm{EF}$ and/or equal to the contralateral undilated renal unit, the dilatation seen does not represent a functionally significant obstructive uropathy. Nevertheless, at present the EF cannot be considered an ideal test, as it is not without drawbacks. There is a variability of $\approx 10 \%$ between the $\mathrm{EF}$ and GFR. Moreover, the values for percentage 
function between ages 1 and 2 years have been defined incompletely. Finally, the EF of the solitary kidney has not been determined completely but it appears to be $\approx 5 \%$ [12].

\section{The Whitaker test}

Because it is invasive and has potential sources of error, the Whitaker test is currently rarely used in the diagnosis of infants with suspected obstruction. When an obstruction is present, the intrapelvic pressure will not increase until after the collecting system is filled completely. Thus, the potential for recording a falsely low pressure exists if there is leakage of fluid outside the collecting system or if the study is terminated before the pelvicalyceal system is filled completely. Another source of error is the intrinsic urine output of the kidney, as it contributes an unknown volume to the total amount of fluid being perfused; the potential for false-positive results should be considered particularly in neonates, as the urine output of the kidney being studied is high.

\section{VCUG}

There has been some controversy over which neonates with a diagnosis of in utero hydronephrosis should undergo VCUG. A common practice is that if the fetal renal pelvic diameter is $>8 \mathrm{~mm}$, and caliectasis or ureterectasis is present, then a VCUG is recommended. Other investigators perform VCUG even if postnatal US shows apparent resolution of the fetal hydronephrosis [45]. A more difficult question is whether to perform VCUG on all infants with any degree of pelvic dilatation [26]. Hollowell et al. [46] reported a coexistence of PUJ obstruction and VUR in 17 (14\%) of 147 consecutive patients undergoing pyeloplasty. Therefore, they recommended that VCUG be part of the routine evaluation of children with suspected PUJ obstruction.

\section{Postnatal treatment}

\section{PUJ obstruction}

The therapeutic management strategies for neonates with such a condition is currently more diverse than ever. Several published series have enthusiastically supported immediate pyeloplasty [47-49]. The rationale underlying this approach is that when obstruction in the neonatal period led to a measured decrease in renal function, some of this was not recoverable by relieving the obstruction. However, in this approach the authors were criticised for having largely relied on drainage curves after frusemide or on IVU to conclude that the systems were irreversibly obstructed. Moreover, it was not known whether the improvement in renal function shown by follow-up renography was caused by early pyeloplasty or to the natural increase in renal function in early postnatal life.

In 1985 there was a major change in the policy outlining the management of antenatally detected PUJ obstruction, after the report of Ransley and Manzoni [41]. They assumed that a decrease in renal function determines the presence of an obstruction that requires surgical intervention. The philosophy underlying this approach is that in the presence of significant obstruction, the filtration of a kidney will ultimately be affected and conversely, in the absence of continuing obstruction, function will remain stable indefinitely. Reports since 1985 emphasize that kidneys can be safely observed if the renal function is initially $>35 \%$ $[42,43,50-52]$. Nevertheless, what remains unknown is the extent of functional damage caused if the kidney requires a delayed pyeloplasty after a trial of conservative management. There are no clear criteria that can predict early which kidney will eventually require surgical intervention. The follow-up studies by Ransley et al. [42] showed that some mature kidneys did not completely recover their lost function after pyeloplasty. This may reflect a possible delay in relieving the PUJ obstruction once the functional change is established. This view was supported by Subramaniam et al. [53], who recently showed that kidneys treated by delayed pyeloplasty had less improvement in renal function than those treated by early pyeloplasty.

Nevertheless, all studies investigating the role of early surgery are retrospective. Recognition of prospective factors that would justify a selective prophylactic operation is currently without foundation. Some recent reports have suggested that kidneys with a pelvic APD of $>2 \mathrm{~cm}$ should possibly be treated by surgery initially, irrespective of renal function [53,54]. Whether early pyeloplasty will ultimately be of greater benefit can only be determined by a controlled randomized study.

\section{Primary obstructive megaureter}

In this case, impressive dilatation of the distal ureter has not been associated with any detectable loss of renal function and when followed serially the dilatation resolves significantly. Of 34 renal units associated with primary obstructive megaureter detected by prenatal US, only two $(6 \%)$ required an operation for diminishing renal function and most showed decreased dilatation on sequential development [12]. Therefore, surgery in the absence of symptoms is recommended only when the relative renal function is decreased $(<35 \%)$ [7]. 


\section{Other causes}

When a ureterocele is present, the best initial treatment is endoscopic incision. With the diagnosis of an obstructed ectopic ureter there are several options, depending upon the degree of function in the associated renal unit, size of the ureter and presence of VUR. When hydronephrosis is related to a neurogenic bladder or the prune-belly syndrome it is necessary to establish that bladder emptying is adequate and usually surgery is not required. When VUR is present antibiotic prophylaxis is the initial management, while multicystic kidneys do not benefit from antibiotic prophylaxis and are followed by serial US [7]. Management of these other causes of hydronephrosis is discussed in detail elsewhere.

\section{References}

1 Thomas DFM. Fetal uropathy. Br J Urol 1990; 66: 225-9

2 Scott J, Renwick M. Antenatal diagnosis of congenital abnormalities in the urinary tract. Br J Urol 1988; 62: 295300

3 Scott JE, Renwick M. Urological anomalies in the Northern Region Fetal Abnormality Survey. Arch Dis Child 1993; 68: $22-6$

4 Livera LN, Brookfield DS, Eggenton JA, Hawnaur JM. Antenatal ultrasonography to detect fetal renal abnormalities: a prospective screening programme. Br Med J 1989; 298: 421-3

5 Helin I, Persson P. Prenatal diagnosis of urinary tract abnormalities by ultrasound. Pediatrics 1981; 78: 879-83

6 Mandell J, Blyth BR, Peters CA et al. Structural genitourinary defects detected in utero. Radiology 1991; 178: 193-6

7 Blyth B, Snyder HM, Duckett JW. Antenatal diagnosis and subsequent management of hydronephrosis. J Urol 1993; 149: 693-8

8 Homsy YL, Saad F, Laberge I et al. Transitional hydronephrosis of the newborn and infant. J Urol 1990; 144: 57983

9 Reznik VM, Murphy JL, Mendoza SL et al. Follow-up of infants with obstructive uropathy detected in utero and treated surgically postnatally. J Pediatr Surg 1989; 24: 1289-92

10 Barakat AJ, Butler MC, Cobb CG et al. Reliability of ultrasound in the prenatal diagnosis of urinary tract abnormalities. Pediatr Nephrol 1991; 5: 12-4

11 Mandell J, Peters CA et al. Chapter 52. Perinatal urology. In Walsh PC, Retik AB, Stamey TA, Vaughan ED, eds, Campbell's Urology. 7th edn, Vol 2. Philadelphia: WB Saunders, 1998: 1601-18

12 Keating MA, Escala J, Snyder HM et al. Changing concepts in management of primary obstructive megaureter. J Urol 1989; 142: 636-40

13 Escala JM, Keating MA, Boyd G et al. Development of elastic fibres in the upper urinary tract. J Urol 1989; 141: 969-73

14 Josephson S. Suspected pyelo-ureteral junction obstruction in the fetus: when to do what? II. Experimental viewpoints. Eur Urol 1991; 19: 132-8

15 Taki M, Goldsmith DI, Spitzer A. Impact of age on effects of ureteral obstruction on renal function. Kidney Int 1983; 24: 602

16 Chevalier RL, Gomez RA, Jones CE. Developmental determinants of recovery after relief of partial ureteral obstruction. Kidney Int 1988; 33: 775-81

17 Chevalier RL, Thornhill BA, Wolstenholme JT, Kim A. Unilateral ureteral obstruction in early development alters renal growth: dependence on the duration of obstruction. J Urol 1999; 161: 309-13

18 Ewigman BG, Crane JP, Frigoletto FD et al. Effect of prenatal ultrasound screening on prenatal outcome. RADIUS study group. N Engl J Med 1993; 329: 821-7

19 Broecker BH, Redwine FO, Peters RE. Reversal of acute polyhydramnios after fetal decompression. Urology 1988; 31: $60-2$

20 Keirse MJ, Meerman MB. Antenatal diagnosis of Potter syndrome. Obstet Gynecol 1978; 52: 645-8

21 Petrikovsky BM, Nardi DA, Radis JF, Hoegsberg B. Elevated maternal alpha-feto protein and mild fetal uropathy. Obstet Gynecol 1991; 78: 262-4

22 Siemens DR, Prouse KA, MacNeily AE, Sauerbrei EE. Antenatal hydronephrosis: Thresholds of renal pelvic diameter to predict insignificant postnatal pelviectasis. Tech Urol 1998; 4: 198-201

23 Grignon A, Filion R, Filiatrault D et al. Urinary tract dilatation in utero: Classification and clinical applications. Radiology 1986; 160: 645-7

24 Zerin JM, Ritchey ML, Chang AC. Incidental vesicoureteral reflux in neonates with antenatally detected hydronephrosis and other renal abnormalities. Radiology 1993; 187: 15760

25 Coret A, Morag B, Katz M et al. The impact of fetal screening on indications for cystourethrography in infants. Pediatr Radiol 1994; 24: 516-8

26 Marra G, Barbieri G, Moioli C et al. Mild fetal hydronephrosis indicating vesicoureteral reflux. Arch Dis Child 1994; 70: 147-50

27 Mandell J, Lebowitz RL, Peters CA et al. Prenatal diagnosis of the megacystis-megaureter association. J Urol 1992; 148: 1487-9

28 Winters WD, Lebowitz RL. Importance of prenatal detection of hydronephrosis of the upper pole. Am J Roentgenol 1990; 155: 125-9

29 Estroff JA, Mandell J, Benacerraf BR. Increased renal parenchymal echogenicity in the fetus: importance and clinical outcome. Radiology 1991; 181: 135-9

30 Vates TS, Johnson MP, Evans MI et al. The use of urinary epidermal growth factor to predict outcomes in fetal obstructive uropathy. AUA Meeting 1997

31 Longaker MT, Golbus MS, Filly RA et al. Maternal outcome after open fetal surgery: a review of the first 17 human cases. JAMA 1991; 265: 737-41

32 Dejeter SW, Gibbons MD. The fate of infant kidneys with fetal hydronephrosis but initially normal postnatal sonography. J Urol 1989; 142: 661-2 
33 Koff SA, Peller PA, Young DC, Pollifrone DL. The assessment of obstruction in the newborn with unilateral hydronephrosis by measuring the size of the opposite kidney. J Urol 1994; 152: 596-9

34 Platt JF, Rubin JM, Ellis HM, DiPietro MA. Duplex Doppler US of the kidney: differentiation of obstructive from nonobstructive dilatation. Radiology 1989; 171: 515-7

35 Bude RO, DiPietro MA, Platt JF et al. Age dependency of the renal resistive index in healthy children. Radiology 1992; 184: 469-73

36 Shokeir AA, Provoost AP, El-Azab M et al. Renal Doppler ultrasound in children with normal upper urinary tracts: Effect of fasting, hydration with normal saline, and furosemide administration. Urology 1996; 47: 740-4

37 Shokeir AA, Provoost AP, El-Azab M et al. Renal Doppler ultrasound in children with obstructive uropathy: effect of intravenous normal saline fluid load and furosemide. J Urol 1996; 156: 1455-8

38 Shokeir AA, Provoost AP, El-Azab M et al. Renal Doppler ultrasound in children with equivocal obstructive uropathy: effect of intravenous normal saline fluid load and frusemide. Br J Urol 1997; 80: 313-8

39 Kass EJ, Fink- Bennett D. Contemporary techniques for the radioisotopic evaluation of the dilated urinary tract. Urol Clin N Am 1990; 17: 273-89

40 Cartwright PC, Duckett JW, Keating MA et al. Managing apparent ureteropelvic junction obstruction in the newborn. J Urol 1992; 148: 1224-8

41 Ransley P, Manzoni G. Extended role of DTPA scan in assessing function and UPJ obstruction in neonate. Dial Ped Urol 1985; 8: 6-8

42 Ransley P, Dhillon H, Gordon I et al. The postnatal management of hydronephrosis diagnosed by prenatal ultrasound. J Urol 1990; 144: 584-7

43 Koff SA, Campbell K. Nonoperative management of unilateral neonatal hydronephrosis. J Urol 1992; 148: 525-31

44 Sukhai RN, Kooy PPM, Wolff ED et al. Evaluation of obstructive uropathy in children. 99mTc-DTPA renography studies under conditions of maximal diuresis. $\mathrm{Br} J$ Urol 1985; 57: 124-9

45 Zerin JM, Richey ML, Chang AC. Incidental vesicoureteral reflux in neonates with antenatally detected hydronephrosis and other renal abnormalities. Radiology 1993; 187: 157-9

46 Hollowell JG, Altman HG, Snyder HM, Duckett JW. Coexisting ureteropelvic junction obstruction and vesicoureteral reflux: diagnostic and therapeutic implications. J Urol 1989; 142: 490-3

47 Bernstein G, Mandell J, Lebowitz R et al. Ureteropelvic junction obstruction in the neonate. J Urol 1988; 140: 1216-21

48 King I, Coughlin P, Bloch EC et al. The case for immediate pyeloplasty in the neonate with ureteropelvic junction obstruction. J Urol 1984; 132: 725-8

49 Guys I, Borella F, Monfort G. Ureteropelvic junction obstruction: prenatal diagnosis and neonatal surgery in 47 cases. J Pediatr Surg 1988; 23: 156-8

50 Arnold AJ, Rickwood AMK. Natural history of pelviureteric obstructions detected by prenatal sonography. Br J Urol 1990; 65: 91-6

51 Madden NP, Thomas DFM, Gordon AC et al. Antenatally detected pelviureteric junction obstruction. Is non-operation safe? Br J Urol 1991; 68: 305-10

52 Sheldon CA, Duckett JW, Snyder HM. Evolution in the management of infant pyeloplasty. J Pediatr Surg 1992; 27: $501-5$

53 Subramaniam R, Kouriefs C, Dickson AP. Antenatally detected pelvi-ureteric junction obstruction: Concerns about conservative management. BJU Int 1999; 84: 335-8

54 Elder JS. Antenatal hydronephrosis. Fetal and neonatal management. Pediatr Clin North Am 1997; 44: 55

\section{Authors}

A.A. Shokeir, MD, PhD, Associate Professor.

R.J.M. Nijman, MD, PhD, Associate Professor.

Correspondence: A. A. Shokeir, Associate Professor of Urology, New Jeddah Clinic Hospital, PO Box 7692, 21472 Jeddah, Saudi Arabia. 